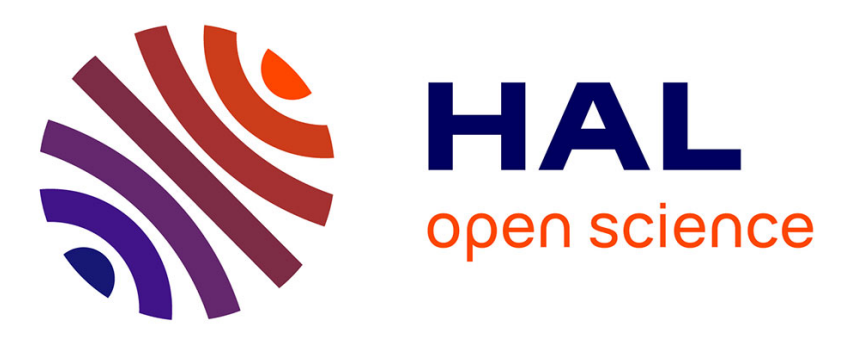

\title{
Bubble splitting in oscillatory flows on ground and in reduced gravity
}

\author{
Harunori Yoshikawa, Farzam Zoueshtiagh, Hervé Caps, Pascal Kurowski, \\ Philippe Petitjeans
}

\section{- To cite this version:}

Harunori Yoshikawa, Farzam Zoueshtiagh, Hervé Caps, Pascal Kurowski, Philippe Petitjeans. Bubble splitting in oscillatory flows on ground and in reduced gravity. European Physical Journal E: Soft matter and biological physics, 2010, 31, pp.191-199. 10.1140/epje/i2010-10561-y . hal-00907768

\section{HAL Id: hal-00907768 \\ https://hal.science/hal-00907768}

Submitted on 21 Nov 2013

HAL is a multi-disciplinary open access archive for the deposit and dissemination of scientific research documents, whether they are published or not. The documents may come from teaching and research institutions in France or abroad, or from public or private research centers.
L'archive ouverte pluridisciplinaire HAL, est destinée au dépôt et à la diffusion de documents scientifiques de niveau recherche, publiés ou non, émanant des établissements d'enseignement et de recherche français ou étrangers, des laboratoires publics ou privés. 


\title{
Bubble splitting in oscillatory flows on ground and in reduced gravity
}

\author{
Harunori N. Yoshikawa ${ }^{1}$ a Farzam Zoueshtiagh $^{2}$ b , Hervé Caps $^{3}$ c, Pascal Kurowski $^{1}$, and Philippe Petitjeans ${ }^{1}$ \\ 1 Physique et Mécanique des Milieux Hétérogène - UMR 7636, 10 rue Vauquelin, 75231 Paris Cedex 05, France \\ 2 Institut d'Electronique, de Microélectronique et de Nanotechnologie - UMR 8520, Avenue Poincaré - BP 60069, 59652 Vil- \\ leneuve d'Ascq Cedex, France \\ 3 GRASP - Optofluidics, Université de Liège, 4000 Liège, Belgium
}

Received: $x x x x$ / Revised version: $x x x x$

\begin{abstract}
The stability of centimeter scale air bubbles is studied in quiescent suspending liquid under imposed oscillatory acceleration field. Experiments were performed in reduced and normal gravity environments. A strong acceleration resulted in an instability leading to the breakups of the bubbles in both gravity environments. The breakup onset was investigated and found to be characterized by a critical acceleration $a_{c r}$. The influence of the liquid viscosity and the gravitational environment was studied. Empirical correlations for the onset are presented and discussed with the intention to reveal splitting mechanism. The inertial mechanism often deemed to cause the breakup of drops subjected to a rapid gas stream is shown to give explanations consistent with the experiments. A breakup criterion for both gravitational environments is proposed through discussions from an energetic point of view.
\end{abstract}

PACS. 47.55.dd Bubble dynamics - 47.35.Pq Capillary waves - 47.20.Dr Surface-tension-driven instability

\section{Introduction}

Flows including dispersed phase are ubiquitous in the nature and over the whole human activity. Blood circulation transporting cells [1], atmosphere with falling raindrops [2], gas-liquid mixture in bubble column reactors and flows in rivers and sea with sand suspension [3] are only some examples of such flows. Behaviors of dispersed phase in different flows have been extensively studied as it is a key for understanding the global transfer of momentum, heat and mass. The size distribution of that phase which decides the dynamics and the associated surface area is fundamental for modeling the transfer mechanism in the flow. The distribution can vary through breakups and/or coalescences due to interactions with continuous phase. The stability of the dispersed phases is thus of great importance.

Fluid systems subjected to periodic excitations have been studied with interest in striking modification of their behavior by the amplitude and frequency adjustments [4]. Faraday instability is one of the well-known subjects of these studies $[5,6]$. Such systems are also investigated with the intention of flow control, e.g., $[7,8]$ and drop atomization $[9,10]$.

\footnotetext{
a Present address: Laboratoire J. A. Dieudonné, Université de Nice Sophia Antipolis, Parc Valrose, 06108 Nice Cedex 2. e-mail: harunori@unice.fr

b e-mail: farzam.zoueshtiagh@univ-lille1.fr

c e-mail: herve.caps@ulg.ac.be
}

Motion of bubbles in vertically oscillating liquid has been a subject of study for performance improvement of bubble column reactors in the process industries [11]. Bubble volume change caused by bubble oscillatory vertical displacement of typical frequency of the order of $100 \mathrm{~Hz}$ can yield a net vertical force component which modifies the bubble rise in the reactors [12]. The present work concerns bubbles in liquid vertically vibrated at frequency of the order of $10 \mathrm{~Hz}$. Behavior of bubbles without volume change in the acceleration field induced by the vibration is studied with focus on vibration-induced bubble breakups. This breaking method can be applied to many industrial processes for controlling bubble sizes. In particular, applications are expected in factories in space, where bubble size is no longer limited to the capillary length scale because of the absence of gravity.

Bubbles subjected to an oscillatory acceleration field are set in translational motion in stagnant suspending liquid by the buoyancy due to the acceleration field. They can eventually break up into smaller bubbles when the field is strong enough. The stability of large bubbles in such a configuration was first experimentally studied by Zoueshtiagh et al. [13]. The oscillatory acceleration field was induced by oscillating a test cell vertically with a given amplitude $A$ and frequency $f$. The authors reported on behavior of bubbles of volume $V_{b}$ ranging from $1.1 \mathrm{~cm}^{3}$ to $8.1 \mathrm{~cm}^{3}$ in various suspending liquids with different surface tensions and viscosities. It was found that the imposed acceler- 
Table 1. Physical properties of the considered liquids.

\begin{tabular}{cccc}
\hline \hline Liquid & $\begin{array}{c}\text { Density } \\
\left(\mathrm{g} / \mathrm{cm}^{3}\right)\end{array}$ & $\begin{array}{c}\text { Viscosity } \\
\left(\mathrm{cm}^{2} / \mathrm{s}\right)\end{array}$ & $\begin{array}{c}\text { Surface tension } \\
(\mathrm{dyn} / \mathrm{cm})\end{array}$ \\
\hline Water & 1 & 0.01 & 72 \\
\hline \multirow{4}{*}{ Silicone oils } & 0.816 & 0.01 & 17.4 \\
& 0.93 & 0.1 & 20.1 \\
& 0.95 & 0.2 & 20.6 \\
& 0.959 & 0.5 & 20.7 \\
\hline \hline
\end{tabular}

ation could have a control over bubble size distribution through bubble breakups. For large $A$ values, compared to the volume equivalent diameter $D_{e}=\left(6 V_{b} / \pi\right)^{1 / 3}$, the bubble size was shown to be limited by twice an effective capillary length, based on the imposed acceleration, $l_{c}=\sqrt{\gamma / \rho A \omega^{2}}(\gamma$ : surface tension, $\rho$ : density of the suspending liquid, $\omega$ : angular frequency).

The purpose of the present work is to better understand the physical mechanisms behind the breakup instability of large bubbles in such oscillatory flows. Experiments have been performed to complete the results presented in the preceding work $[13,14]$. Particularly, the influence of a solid wall have been considered, by experiments on ground in normal terrestrial gravity environment (later designated by $G$ ) where bubbles were always in the proximity of a container wall, as well as in a microgravity environment (designated by $\mu G$ ), where the bubbles were apart from any walls. A universal criterion for both gravity environments will be proposed.

\section{Experimental set-ups and Control Parameters}

Experiments were prepared with the intention to determine the regimes for which a centimetric bubble subjected to vertical vibrations becomes unstable and breaks up. For this purpose, a test cell, which consisted of a polycarbonate parallelepiped container of $6.0 \times 8.0 \times 6.0 \mathrm{~cm}^{3}$ filled by a test liquid and an air bubble of a given volume, was set in vertical oscillation. The liquid was either deionized water or various silicone oils. The physical properties of those fluids are listed in Table 1 . The cell was prepared by filling the container through a $0.2 \mathrm{~cm}$ diameter side hole. The container was first fully filled with liquid and then some of the liquid was removed by a graduated syringe to create a bubble of a controlled volume. The hole was then blocked with a screw having the same length as the wall thickness to seal the cell. The tested bubble volume $V_{b}$ ranged between $2 \mathrm{~cm}^{3}$ and $7 \mathrm{~cm}^{3}$.

For exploring the excitation frequency-amplitude plane, two distinct set-ups have been used. Each of these was related to a particular range of frequency and amplitude. The first setup (ST1) produced large oscillation amplitudes $(0.75 \leq A \leq 12 \mathrm{~cm})$ but delivered low oscillation frequencies $(0.1 \mathrm{~Hz} \leq f \leq 10 \mathrm{~Hz})$, while the second setup

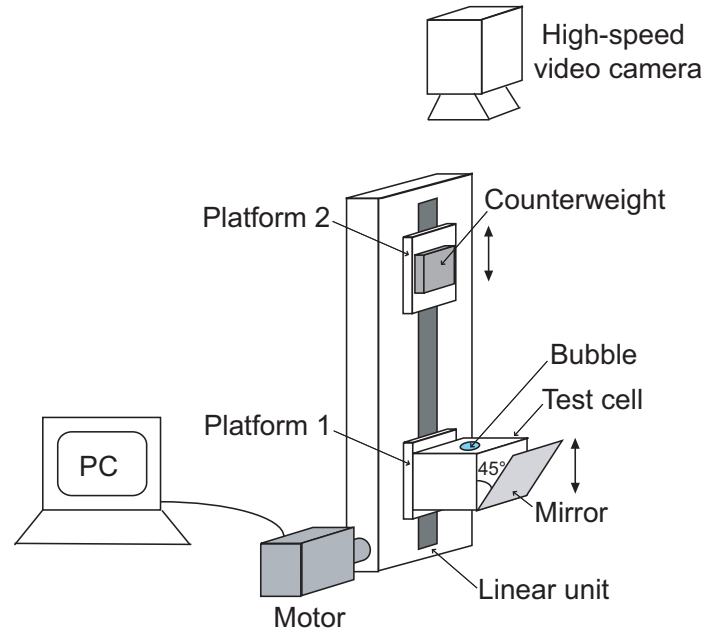

Fig. 1. Illustration of the first experimental set-up (ST1) for low frequency experiments used in micro and normal gravity environments.

(ST2) provided small amplitudes $(A \leq 1 \mathrm{~cm})$ at much larger frequencies $(5 \mathrm{~Hz} \leq f \leq 100 \mathrm{~Hz})$.

The setup ST1 is shown in fig. 1. It consisted of a linear unit system connected to a SGMPH-04AA Fenwick servomotor. The system converted the rotating motion of the drive pin into a linear movement of the guide platforms 1 and 2. The test cell was attached to the lower sliding plate, i.e. platform 1 , while on platform 2 a counterweight of the cell was mounted. The platforms were moved to the middle or apart simultaneously. This anti-phase movement was aimed at reducing vibration transmission to the frame structure of the setup. The motion of the drive was computer controlled. The accuracy of the amplitudes and frequencies were held to within $\pm 0.1 \mathrm{~cm}$ and $\pm 1 / 60 \mathrm{~Hz}$, respectively. The drive motion, hence the movement of the unit, could provide sinusoidal motions at speeds up to $500 \mathrm{~cm} / \mathrm{s}$ and accelerations up to $\sim 3500 \mathrm{~cm} / \mathrm{s}^{2}$.

The setup ST2 consisted of a commercial electromagnetic shaker providing vertical vibrations to the test cell. It was driven by amplified electric signals of a function generator. An accelerometer attached to the cell measured the provided accelerations. The acceleration values between 800 and $10000 \mathrm{~cm} / \mathrm{s}^{2}$.

Experiments in $G$ were performed in the laboratory with either ST1 or ST2 . Bubbles of different volumes $(2 \leq$ $\left.V_{b} \leq 7 \mathrm{~cm}^{3}\right)$ in different test liquids were subjected to acceleration of widely ranged frequency $(0.1 \leq f \leq 100 \mathrm{~Hz})$. Experiments in $\mu G$ were realized on aircraft in parabolic flights. At each parabola, microgravity state lasted $22 \mathrm{~s}$ and its quality was typically $10^{-2} \mathrm{~g}\left(\mathrm{~g}=981 \mathrm{~cm} / \mathrm{s}^{2}\right)$. Bubbles of $3 \mathrm{~cm}^{3}$ were accelerated in different test liquids by ST1.

Bubble behavior was observed from above with a digital high-speed video camera at typically 150 images per second with an exposure time of typically $1 \mathrm{~ms}$. The optical axis of the camera was set parallel to the translation 


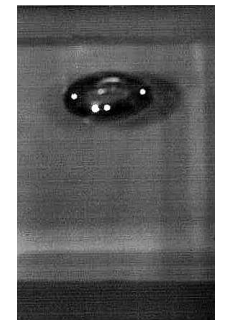

$t=0 \mathrm{~ms}$

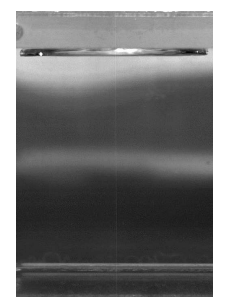

$t=0 \mathrm{~ms}$

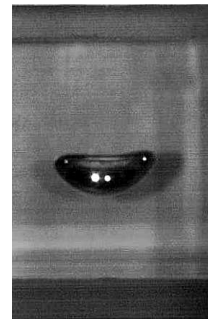

$225 \mathrm{~ms}$

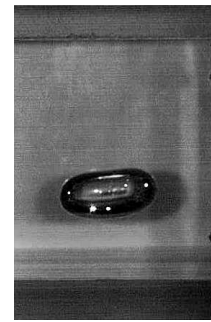

$361 \mathrm{~ms}$

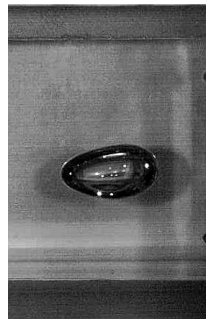

$482 \mathrm{~ms}$

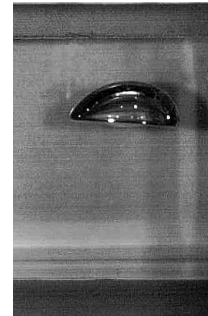

$650 \mathrm{~ms}$

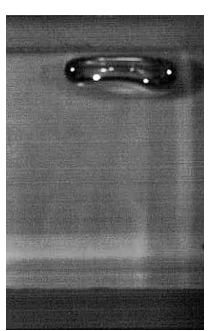

$851 \mathrm{~ms}$

(a) Microgravity (silicone oil of $0.5 \mathrm{~cm}^{2} / \mathrm{s}, A=4 \mathrm{~cm}$ and $f=1 \mathrm{~Hz}$ )

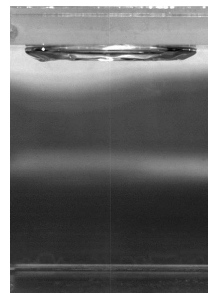

$41 \mathrm{~ms}$

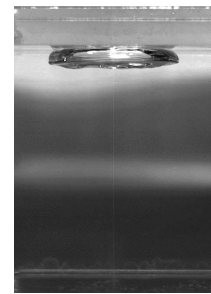

$97 \mathrm{~ms}$

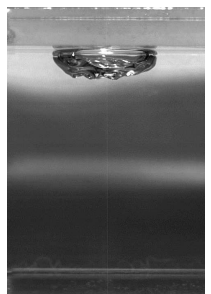

$164 \mathrm{~ms}$

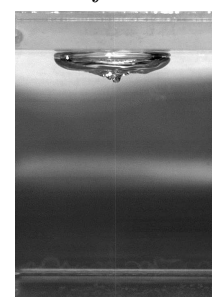

$200 \mathrm{~ms}$

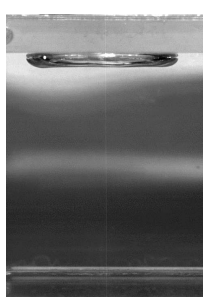

$245 \mathrm{~ms}$

(b) Normal gravity (silicone oil of $0.01 \mathrm{~cm}^{2} / \mathrm{s}, A=2.0 \mathrm{~cm}$ and $f=2.8 \mathrm{~Hz}$ )

Fig. 2. Side views of bubble motion under oscillatory acceleration field.

direction of the cell so that the bubble motion could be observed in a longitudinal view from the top. Side view of the cells was allowed by a 45 degree inclined mirror attached to the cell (see fig. 1). Thanks to the mirror, longitudinal and transversal views of the cell were simultaneously recorded on the same pictures.

\section{Dimensionless numbers}

Various time scales can be associated with bubble dynamics in present experiments. Besides the time scale of the oscillations $t_{\text {osci }} \sim \omega^{-1}$, the viscous time $t_{\nu} \sim D_{e}^{2} / \nu$ and the advection time $t_{a d v} \sim D_{e} / A \omega$ characterise the flow surrounding the bubble. Bubble's free oscillation is characterised by the capillary time $t_{c} \sim\left(\rho D_{e}^{3} / \gamma\right)^{1 / 2}$. These four time scales yield the following three dimensionless parameters: the dimensionless frequency $\Omega=t_{\nu} / t_{\text {osci }}$, the dimensionless amplitude $K_{C}=t_{a d v} / t_{\text {osci }}$ that is identical to the Keulegan-Carpenter number and the Ohnesorge number $O h=t_{c} / t_{\nu}$. More explicitly, they are defined as:

$$
\Omega=\frac{\omega D_{e}^{2}}{2 \nu}, \quad K_{C}=\frac{2 A}{D_{e}} \quad \text { and } \quad O h=\sqrt{\frac{\rho \nu^{2}}{D_{e} \gamma}}
$$

In the present experiments, the dimensionless amplitude $K_{C}$ was varied over a wide range from 0.018 to 14 . The Ohnesorge number $O h$ was also varied widely but always had values smaller than unity, ranging from $8.8 \times 10^{-4}$ to 0.16 . Regarding the frequency parameter $\Omega$, its values were much larger than the unity in both $G$ and $\mu G$ when bubble breakups were observed $\left(19<\Omega<5 \times 10^{4}\right.$ in $G$ and $12<\Omega<2600$ in $\mu G)$. These ranges of $O h$ and $\Omega$ imply that viscous dissipation occurs slowly compared to the oscillation and capillary time scales. Furthermore, the bubble Reynolds number $R e_{b}=U_{b} D_{e} / \nu$ can be estimated by $R e_{b} \sim K_{C} \Omega$ with the bubble velocity $U_{b} \sim A \omega$. It is found that $R e_{b}$ is larger than 200 for the majority of experiments.

\section{Experimental Results}

\subsection{Bubble shape \& motion}

\subsubsection{In micro gravity, $\mu G$}

In $\mu G$, the cell's oscillatory translation, $-A \cos \omega t$, imposed an unsteady acceleration to the system. The resulting artificial buoyancy $\rho V_{b} A \omega^{2} \cos \omega t$ set the bubble into motion. For small amplitude and frequency values, the bubble was animated by an up-and-down motion with the imposed oscillation frequency without breaking (see fig. 2 (a)). As $A$ was increased, the amplitude of the bubble translational motion, $A_{b}$, was also increased until a saturation caused by the cell's finite hight. During the translational motion, the bubble exhibited different shapes, from an ellipsoid to a spherical cap. For the tested experimental parameters, the observed bubble shapes are in agreement with dynamical regimes reported for buoyancy-driven ascending bubbles in a stagnant liquid [15].

The amplitude $A_{b}$ and phase $\theta$ of the bubble's relative motion to the cell have been measured by processing space-time diagrams representing translational motion of the bubble. Figure 3 shows a typical example of such diagrams. As shown by the smaller image in the figure, a narrow band of image passing through the bubble center is extracted along the direction of bubble's principle motion. These bands of image at different instants are arranged chronologically to construct a space-time diagram 


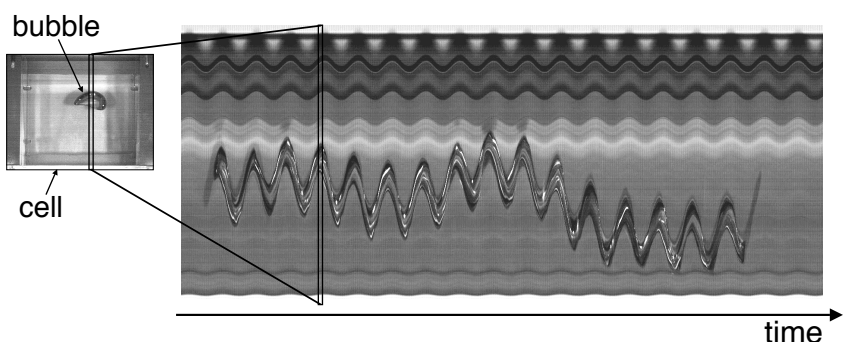

Fig. 3. Space-time diagram of bubble motion (the right larger image) in transversal view in $\mu G$. The suspending liquid is silicone oil of $0.5 \mathrm{~cm}^{2} / \mathrm{s}$ and the oscillation parameters are $A=1.5 \mathrm{~cm}$ and $f=1.6 \mathrm{~Hz}$. The left smaller image shows how the diagram is constructed (see the text for details).

(the larger image in the figure). The oscillatory trajectory at the middle of the diagram represents the bubble motion, while the sinusoidal waves at upper and lower ends show cell's motion. It is seen that the bubble motion is sinusoidal and has well-defined amplitude and phase. Measured values of $A_{b}$ scaled by the amplitude of the cell's motion $A$ and the phase $\theta$ are shown in fig. 4 (a) and (b), respectively. The ratio $A_{b} / A$ is seen to group around a single curve while plotted as a function of the dimensionless amplitude $K_{C}$. For large $K_{C}$ values, it appears that the experimental bubble amplitude $A_{b}$ is independent of the frequency $\Omega$. Regarding the phase shown in fig. 4 (b), the bubble motion always precedes the cell's motion. This arises from the liquid phase movement delayed by its inertia. Hence, the bubble motion is in advanced phase. The data also exhibit increasing tendencies with $K_{C}$ towards $\theta=\pi / 2$. Most of the experimentally determined phase values are beyond the maximum phase advance $(\sim \pi / 8)$ that a bubble can attain at small Reynolds numbers ( $c f$. fig. 11.15 of Clift et al. [15]). This observation suggests that bubble motions in present experiments are associated with large Reynolds numbers.

As seen in figure 2 (a), bubbles undergo large deformation during their oscillatory translation. This deformation will particularly act upon the bubble's associated drag coefficient and the resulting motion. Therefore, the amplitude $A_{b} / A$ and the phase $\theta$ should depend on the Ohnesorge number $O h$ to reflect the capillary effects. In order to investigate the influence of $O h$, a least square fit of the power law $\Omega^{q} O h^{r} K_{C}^{s}$ was applied to the experimental data on $A_{b} / A$. It was found that $q=-0.002, r=-0.150$ and $s=-0.517$. The small value of $q$ implies independency of the oscillation amplitude from $\Omega$. The value of $s$ nearly equal to -0.5 suggests that the bubble velocity $U_{b}\left(=A_{b} \omega\right)$ is scaled by $\left(A \omega^{2} D_{e}\right)^{1 / 2}$, as spherical cap bubbles rising in stagnant liquid [16]. Based on these observations, a power law $A_{b} / A \sim O h^{r^{\prime}} K_{C}^{-0.5}$ is proposed and the value of $r^{\prime}$ is derived from a least square fit to the data. It is found that:

$$
A_{b} / A=0.59 O h^{-0.15} K_{C}^{-0.5}
$$

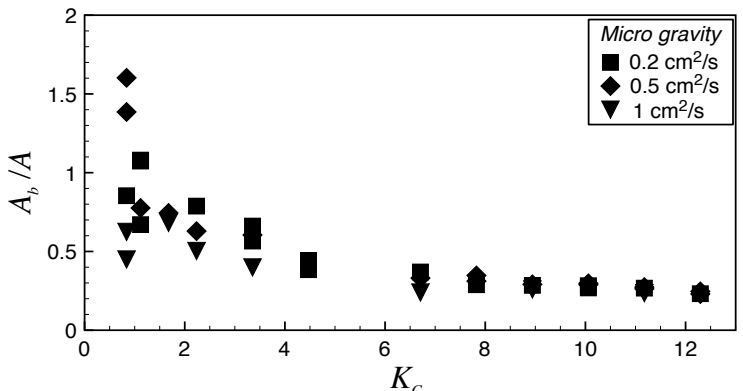

(a)

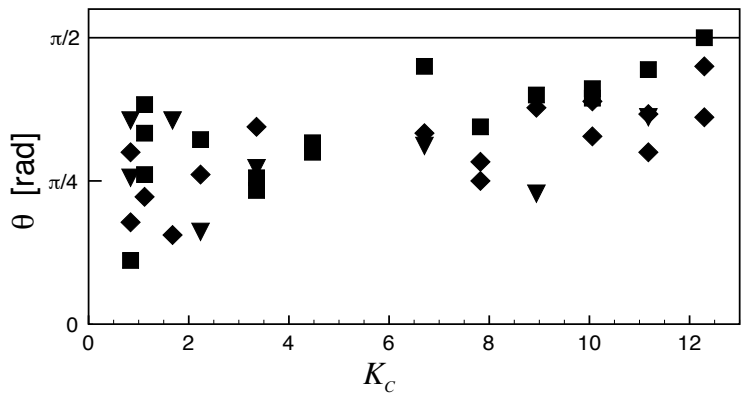

(b)

Fig. 4. Amplitude and phase of bubble motion in $\mu G$.

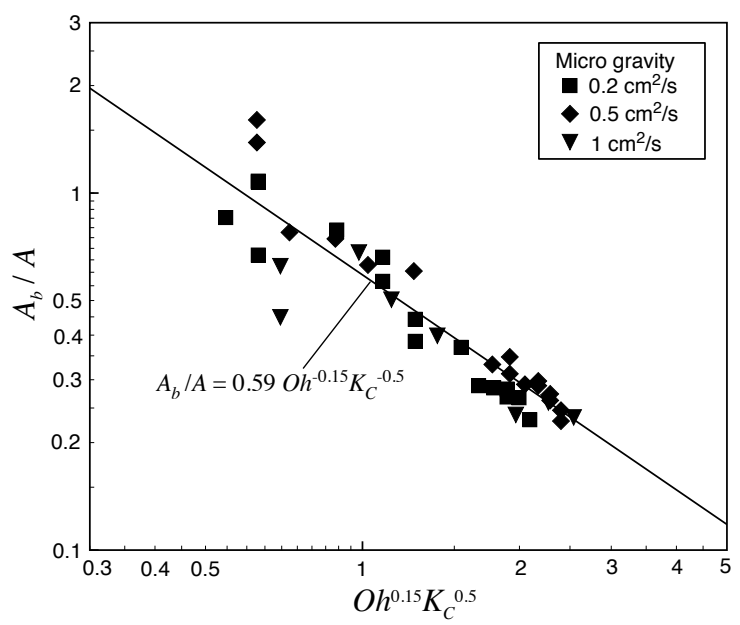

Fig. 5. Correlation for the amplitude $A_{b}$ of the bubble motion

It appears that the above relation correlates well all the experimental data (see fig. 5 ). The bubble velocity $U_{b}$ is then calculated as $U_{b}=A_{b} \omega=0.59 O h^{-0.15} K_{C}^{-0.5} A \omega$. Use of the definition of $K_{C}$ (1) in this equation leads to the following expression of $U_{b}$ :

$$
U_{b}=\frac{0.416}{O h^{0.15}} \sqrt{A \omega^{2} D_{e}}
$$

This velocity is identical to the terminal velocity of a spherical cap bubble determined by Davies and Taylor [16] but with a corrective factor proportional to $O h^{-0.15}$. This correction represents the influence of bubble deformation during the oscillatory translational motion. 


\subsubsection{In normal gravity, $G$}

In $G$ environment, prior to the experiments the bubble lies below the upper wall due to buoyancy. It has a disklike shape of a thickness twice the capillary length $\ell_{c 0}=$ $(\gamma / \rho g)^{1 / 2}$ because of its large volume compared to the capillary length $\left(D_{e}>\ell_{c 0}\right)[17]$. With small acceleration values of the cell, the bubble and the liquid are translated by the cell without any significant relative motion between them. A large acceleration leads to a relative motion between the two phases. The bubble is periodically squeezed on the top wall with the same frequency as the cell's motion without detaching from it (see fig. 2 (b)). Further increase of the acceleration can lead to partial or full detachment of the bubble from the wall and bubble splitting into smaller parts (for more detailed description cf. $[13,18])$.

In high frequency experiments carried out with set-up $\mathrm{ST} 2$, for excitation amplitude $A$ smaller than twice $\ell_{c 0}$, two main differences have been observed in bubble dynamics compared to low frequency experiments (ST1). First, surface waves formed on the bubble lower surface above a certain acceleration value were standing waves and non axisymmetric, while in ST1 experiments they were propagative and axisymmetric (cf. [13]). Second, for large acceleration values, bubble breakups occurred by ejections of smaller bubbles from the mother one rather than the uniform splittings such as typically observed in the low frequency experiments. These phenomenological differences in low and high frequency experiments suggest that experiments carried out with an excitation amplitude $A \lesssim \ell_{c 0}$ fall in different dynamical regime. Standing wave formation in high frequency experiments suggests that Faraday instability probably plays a role in bubble breakups and ejections. The investigation of bubble breakup in such regime is out of scope of the present paper and will not be discussed further.

\subsection{Bubble breakup thresholds}

For large accelerations, bubbles are first pinched at one or more location before breaking into smaller parts. For breakups into more than two parts, splits can either occur simultaneously or successively until all bubbles reached sufficiently small sizes and the system reached a stable state. Breakups following bubble pinching usually happened within $0.1 \mathrm{~s}$.

\subsubsection{Breakup thresholds in micro gravity}

In $\mu G$, the breakups usually occurred apart from the cell walls (see fig. 6). In longitudinal views, the bubble is clearly seen to first deform into a peanut shape before being pinched in the middle and break up. Due to the finite size of the cell, walls could sometimes influence the breakup mechanism. The initial deformation leading to the breaking could occasionally be initiated by an encounter event of the bubble with the wall.
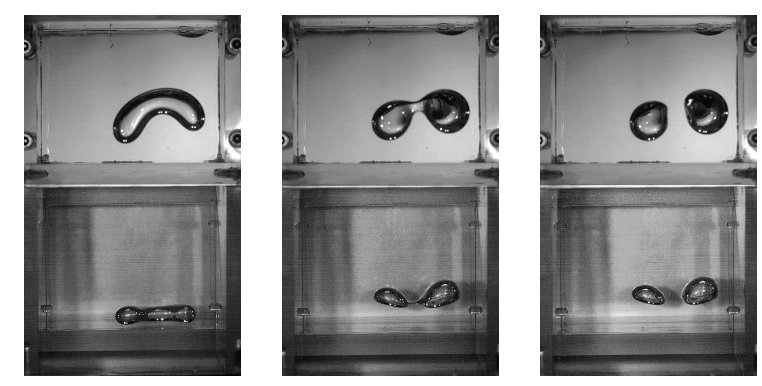

Fig. 6. Bubble at the moment of breakup at the middle of the cell in $\mu G(A=4.2 \mathrm{~cm}$ and $f=1.3 \mathrm{~Hz}$ with silicone oil of $0.5 \mathrm{~cm}^{2} / \mathrm{s}$ ). In each picture, the upper and lower half is in longitudinal and transversal view, respectively.

The critical frequency $f_{c r}$ of the breakup has been determined by varying the frequency $f$ for given amplitude $A$ values. This determination has been made with precisions of typically $\pm 0.05 \mathrm{~Hz}$. Results are presented in fig. 7 for $\mu G$ experiments. A general tendency reveals that the acceleration $a_{c r}=A\left(2 \pi f_{c r}\right)^{2}$ is approximately constant at the thresholds for a given suspending liquid. This finding is consistent with the preceding experiments [13] for ground experiments. However, the critical acceleration values found in $\mu G$ are around four times smaller than those in $G$.

Figure 7 shows that a bubble in water $(\gamma=72 \mathrm{dyn} / \mathrm{cm})$ breaks up at a critical acceleration $a_{c r}=280 \mathrm{~cm} / \mathrm{s}^{2}$ in average, while it breaks at only $a_{c r}=94 \mathrm{~cm} / \mathrm{s}^{2}$ in the silicone oil of $0.01 \mathrm{~cm}^{2} / \mathrm{s}(\gamma=17.4 \mathrm{dyn} / \mathrm{cm})$. This reveals the stabilizing effect of surface tension. Regarding the influence of the suspending liquid viscosity $\nu$, results from different silicone oils with various viscosity values show a stabilizing effect. The critical acceleration $a_{c r}$ is, for example, $394 \mathrm{~cm} / \mathrm{s}^{2}$ in the oil of $1 \mathrm{~cm}^{2} / \mathrm{s}$ that is four times larger than in the oil of $0.01 \mathrm{~cm}^{2} / \mathrm{s}$. The data for all the suspending liquids were used to find an empirical law of the breakup threshold in terms of the dimensionless parameters $K_{C}, \Omega$ and $O h$. As shown in fig. 8, it is found that all the data of the experimentally determined thresholds group around a single line extending over six orders of magnitude along the abscissa and three orders of magnitude along the ordinate. Data scatter and small deviations observed in the figure come from the difficulties encountered in evaluating the breakup threshold with precision in parabolic flights where $\mu G$ phase could only last 22 second maximum. A least square fit to the data yields:

$$
K_{C} O h^{1.7} \Omega^{1.93}=29.3
$$

It implies that the critical acceleration $a_{c r}$ increases only slightly with the frequency $\left(a_{c r} \propto \Omega^{0.07}\right)$ and has a dependency on the liquid physical properties such that $a_{c r} \propto$ $\rho^{-0.85} \nu^{0.23} \gamma^{0.85}$. 


\subsubsection{Breakup thresholds in normal gravity}

In $G$, the breakups are completed on the top wall. They begin with wave formation on the lower surface. The bubble forms a complete or incomplete torous before its pinchingoff leads to splitting. More details of the breakups in $G$ are available in [13].

Critical frequency values in $G$ experiments are shown in fig. 7 for bubbles of $3 \mathrm{~cm}^{3}$. Stronger oscillations were required to destabilize the bubble in $G$ compared to $\mu G$ experiments. The relation $a_{c r}=c s t$ at the bubble breakup thresholds reported by [13] is confirmed in both set-ups except for the data with frequencies higher than $20 \mathrm{~Hz}$ (correspondingly, $A \lesssim 0.1 \mathrm{~cm}$ ). The latter data will no longer considered in the present work, because these correspond to bubbles in a dynamical regime different from that in low frequency experiments, as described in sect. 4.1.2. The critical acceleration values are much larger than those in $\mu G\left(e . g\right.$. , in the oil of $0.01 \mathrm{~cm}^{2} / \mathrm{s}, a_{c r}=779 \mathrm{~cm} / \mathrm{s}^{2}$ in $G$ while $a_{c r}=94 \mathrm{~cm} / \mathrm{s}^{2}$ in $\mu G$ ) and these values are seen to increase with the viscosity and with the surface tension, as observed in the experiments in $\mu G$. A similar correlation to that for $\mu G$ experimental data was derived for $G$ data using dimensionless parameters $K_{C}, \Omega$ and $O h$. Figure 8 reveals that $G$ data also group around a single line extending over several orders of magnitude along the abscissa and the ordinate. A least square fit to the data yields:

$$
K_{C} O h^{1.7} \Omega^{1.98}=208
$$

It appears, with quite surprise, that this power law is similar to that obtained for $\mu G$ experiments (4) in spite of qualitatively different behavior of bubbles observed in $G$ from in $\mu G$. As described above, wall influence is apparently important in bubble motion and breakup. The essential difference in the power laws of the breakup onset, however, concern only the constants appearing in equations (4) and (5), which is significantly larger in $G$ due to larger $a_{c r}$ values for bubble breaking. This large difference will be explained energeticaly in the discussion of sect. 5.3.

\section{Discussion on the breakup mechanism}

In the literature, a large variety of criteria for the breakups of fluid particles (bubbles and drops) have been proposed under different flow configurations (see review papers, e.g. [19]). The criteria can be classed into three types [20]. (i) For particles in motion with small Reynolds numbers, viscous shear at the surface is responsable for the distortion and breakup of bubbles. Therefore, the criteria are expressed in terms of the capillary number, which reflects the relative importance of viscous to capillary forces. (ii) For particles in motion with large Reynolds numbers, inertia of the surrounding fluid flow is responsible for bubble deformation and breakups. The criteria write in terms of the Weber number, $W e$, that compares the flow inertia with the capillary pressure. (iii) For particles subjected to time periodic excitations of their natural frequency, resonance has been proposed as breakup mechanism. Besides

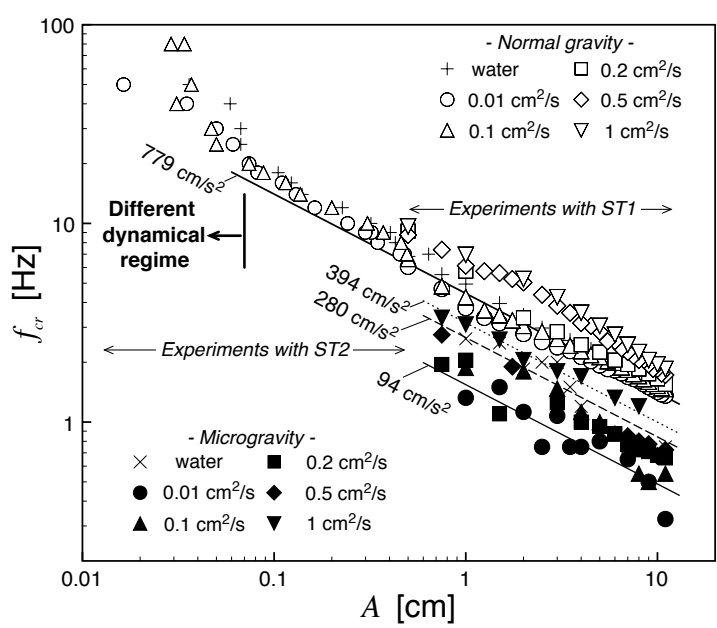

Fig. 7. Critical frequency of bubble breakups in micro and normal gravity environments. Bubble volume is $3 \mathrm{~cm}^{3}$ for all the data. Curves of constant acceleration are also shown for the values discussed in the text.

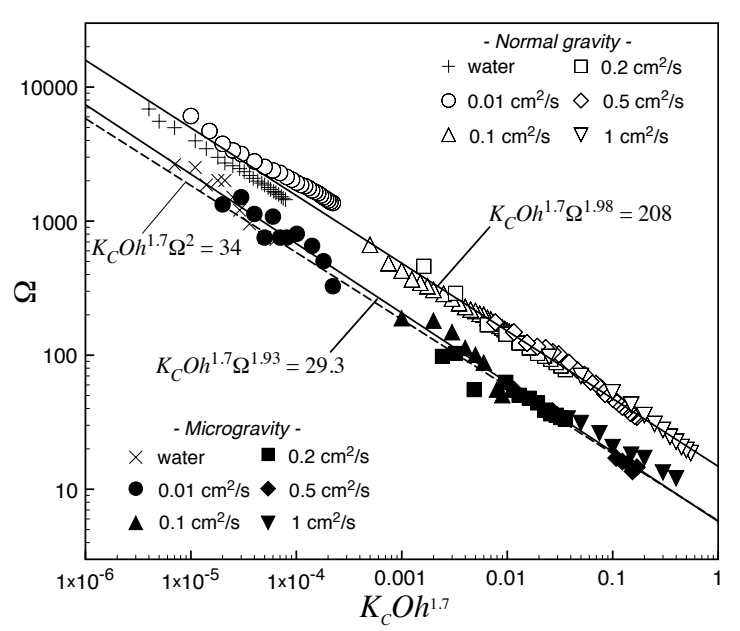

Fig. 8. Correlations of the breakup threshold in micro and normal gravity environments in terms of dimensionless numbers. Bubble volume is $3 \mathrm{~cm}^{3}$ for all the data.

these three classes, the Rayleigh-Taylor instability at the particle surface is often pointed out as destabilising mechanism for particles ascending or descending freely in stagnant suspending phase and for bubbles at the rebound stage of cavitation collapse [21,22]. For understanding the bubble breakups observed in present experiments, we examine the plausible mechanisms among them in this section. The Rayleigh-Taylor, resonance and inertial mechanisms are considered.

\subsection{Mechanism due to the Rayleigh-Taylor instability}

In the present experiments, bubble breakups have occurred at a constant value of the acceleration field for a given suspending liquid. This might suggest that breakups are 
provoked by Rayleigh-Taylor instability as the latter originates in the acceleration, $a$, of the interface between two fluids of different densities. The breakup of fluid particles ascending or descending freely by the buoyancy in stagnant fluid has been studied by models based on this instability [21,23,24]. According to theoretical study of Kitscha \& Kocamustafaogullari [24], the breakup criterion for bubbles in such a configuration writes:

$$
\frac{\rho a D_{e}^{2}}{\gamma}>732.8\left(1+\sqrt[4]{\frac{\rho a D_{e}^{2}}{\gamma}} O h\right)^{1.66}
$$

This model has been compared successfully with experiments when $a$ was equal to the gravitational acceleration $g$. One can apply the model to the case of oscillatory acceleration field in the limit of small frequency; in other words, in the limit of large oscillation amplitude. In $\mu G$ experiments, the interface acceleration $a$ is given by subtracting the acceleration of the bubble motion in the cell's reference frame from the imposed acceleration field. This yields $a=-A \omega^{2} \cos \omega t-A_{b} \omega^{2} \cos (\omega t+\theta)$. The experiments show that $A_{b}$ is small compared to $A$ for large $K_{C}$ values (see fig. $4(\mathrm{a})$ ). The maximum value of $a$ can then be estimated by $A \omega^{2}$. Introducing this estimate into the criterion (6) yields $A \omega^{2}>732.8 \gamma / \rho D_{e}^{2}$ for bubbles with small Ohnesorge numbers (i.e. in liquid of small viscosity). Although this result correlates a breakup at a constant acceleration, a comparison with experiments shows that $a_{c r}$ is largely overestimated. For instance, the model predicts for the silicone oil of $0.01 \mathrm{~cm}^{2} / \mathrm{s}$ a critical acceleration $a_{c r}=4870 \mathrm{~cm} / \mathrm{s}^{2}$ which is, respectively, fifty and seven times larger than the corresponding $a_{c r}$ obtained in experiments in $\mu G$ and $G$. This result suggests that the breakup regime is not governed by a Rayleigh-Taylor instability.

\subsection{Mechanism due to resonance}

Free oscillation of a bubble has natural frequencies $f_{\ell}$ $(\ell=2,3, \cdots)$ scaled by the inverse of the capillary time scale $t_{c}^{-1}=\left(\gamma / \rho D_{e}^{3}\right)^{1 / 2}$. The explicit expression of $f_{\ell}$ for a spherical bubble in inviscid liquid can be found in, e.g. [25]. Viscous correction to it was given by Prosperetti [26] for suspending liquids of any viscosity. For a bubble of volume $3 \mathrm{~cm}^{3}$ in silicone oils of viscosity ranging 0.01 to $1 \mathrm{~cm}^{2} / \mathrm{s}$, the fundamental mode $(\ell=2)$ has natural frequencies ranging from $3.02 \mathrm{~Hz}$ to $1.56 \mathrm{~Hz}$ which are of the same order of magnitude as the imposed frequency in the present experiments. Although in fig. 8 no preferred frequency was observed which could suggest a resonance breakup mechanism, an interaction of the imposed frequencies with bubbles eigen modes was found in $G$ experiments. Figure 9 plots the dimensionless critical acceleration $a_{c r} / G$ for silicone oil of viscosity $0.1 \mathrm{~cm}^{2} / \mathrm{s}$ as a function of the frequency scaled by the natural frequency $f_{2}$ calculated by the viscous theory [26]. In this figure, it is shown that $a_{c r}$ has a minimum when $f / f_{2}$ is equal to 1.5. This behavior of the critical acceleration

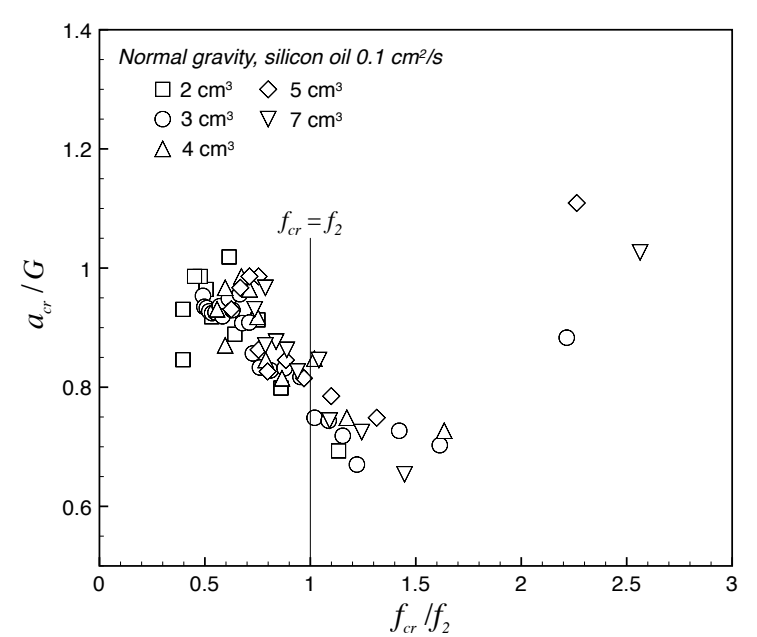

Fig. 9. Dimensionless critical acceleration for the breakups of a bubble of $3 \mathrm{~cm}^{3}$ in silicone oil of viscosity $0.1 \mathrm{~cm}^{2} / \mathrm{s}$. The bubbles of volumes $V_{b}=2,3,4,5$ and $7 \mathrm{~cm}^{3}$ have natural frequencies $f_{2}$ of $3.66,2.99,2.60,2.32$ and $1.97 \mathrm{~Hz}$, respectively.

suggests that the resonance can influence the principle breakup mechanism. This eventual influence of bubble's natural frequency could be explained by wall effects, since no similar finding has been made in $\mu G$. In $G$, bubbles are in contact with the upper container wall. Bubble deformations are required for any relative motion of the bubble to the liquid, while it is not the case in $\mu G$. In turn, perturbation flows destabilizing the bubble are affected by bubble deformations which become the most important with excitations at natural frequency.

\subsection{Inertial mechanism}

According to the results on the bubble velocity amplitude in $\mu G(3)$, the bubble Reynolds number $R e_{b}$ is calculated as $R e_{b}=U_{b} D_{e} / \nu=0.59 O h^{-0.15} \sqrt{K_{C} \Omega^{2}}$. At the breakup onset, this gives typically large values as suggested earlier, except for the oil of $1 \mathrm{~cm}^{2} / \mathrm{s}$. Using the correlation for the onset (4), one can find, e.g., $R e_{b} \sim 2000$ and 50 for the oil of 0.01 and $0.5 \mathrm{~cm}^{2} / \mathrm{s}$, respectively. Hence, the inertial breakup would be one of the most credible mechanisms. Stability studies of drops subjected to air blasts and buoyancy-driven (ascending or descending) drops show the following criterion for inertial breakups in the limit of small viscosity $[27,28]$ :

$$
W e>12
$$

This criterion expressed in terms of the Weber number $W e=\rho U_{b}^{2} D_{e} / \gamma$ means that a pressure perturbation, $\rho U_{b}^{2}$, caused by flow inertia destabilizes drops against the stabilizing capillary pressure, $\gamma / D_{e}$. This could be applied to the present bubbles, when the time scale of oscillation, $t_{\text {osci }}$, remains large compared to the breakup time scale $(\sim 0.1 \mathrm{~s})$ and when the influence of cell's walls is absent (i.e. in $\mu G$ ). By considering the maximum velocity $A_{b} \omega$ 
that a bubble can attain, the criterion (7) can be written for the present experiments as:

$$
W e=\frac{\rho\left(\omega A_{b}\right)^{2} D_{e}}{\gamma}=\left(K_{C} O h \Omega A_{b} / A\right)^{2}>12
$$

where the third term just corresponds to Weber number written as a function of dimensionless quantities $K_{C}, \Omega$, $O h$ and $A_{b} / A$. The latter amplitude ratio $A_{b} / A$ can be estimated from the measurements of bubble translation amplitude. Insertion of the derived empirical law (2), which takes into account bubble deformations, leads to the following breakup criterion:

$$
K_{C} O h^{1.7} \Omega^{2}>34
$$

Comparison of this criterion with the least square fit to the experimental data of eq. (4) shows very good agreement. In fig. 8 the curve of eq. (9) is represented by a dashed line and is seen to fit very well the data obtained in $\mu G$.

\subsection{A common breakup criterion in term of Weber number for both $G$ and $\mu G$ experiments}

Although different empirical laws derived for experiments in $G$ and $\mu G$ were found to be similar (see eqs. (4) and (5)), they did not permit to define a universal breakup criterion for both environments. In this section, following the results from previous section, one such criterion will be proposed.

In $G$ experiments, the bubble lower surface was observed to develop waves prior to its breakup, while its upper surface typically remained in contact with the top wall of the cell (cf. [13]). This suggests the destabilization of the lower surface is necessary for bubble breakups. Hence, flow inertia has to overcome not only the stabilizing effects due to surface tension but also those due to gravity. In other words, the inertial perturbation $\left(\rho U_{b}^{2}\right)$ has to be strong enough to break up bubbles against the capillary pressure $\left(\gamma / D_{e}\right)$ and the hydrostatic pressure due to the gravity $\left(\rho g D_{e}\right)$. Therefore, the criterion (7) should be modified accordingly. At the threshold, the relation $\rho U_{b}^{2} \sim \gamma / D_{e}+\rho g D_{e}$ is expected from an energetic point of view. The universal criterion in a dimensionless form will be written as:

$$
W e>12+C \text { Bo }
$$

where $\mathrm{Bo}=\rho g D_{e}^{2} / \gamma$ is the Bond number and the constant 12 has been chosen to meet the criterion (7) in the limit of $\mu G$. In order to evaluate $W e$, one needs estimates of bubble velocity $U_{b}$ which are difficult to obtain in $G$ as ground experiments are associated with the large bubble deformation near the top wall. Hence, we assume that $U_{b}=\omega A_{b}$ can be evaluated by the same law as that in $\mu G$ (eq. (2)). The latter hypothesis is based on the observed similarity of the experimental breakup criteria (4) and (5). Figure 10 plots $W e$ divided by $12+C$ Bo as a function of $\Omega$. The ajustable parameter $C$ is set to be

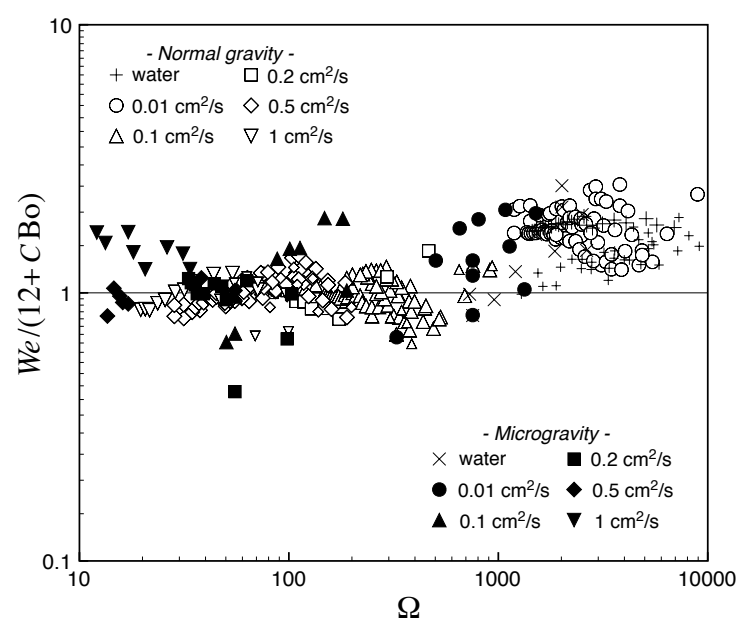

Fig. 10. Critical Weber number values in micro and normal gravity environments.

$1 / 2$ to yield favourable agreements with the experiments. It is seen that the breakup criterion (10) is well satisfied by the experimental data of both environments. This suggests again that observed bubble breakups are caused by the inertial perturbation in suspending fluid. Note that, as the Bond number is relatively large in $G$ experiments $(44 \leq$ Bo $\leq 240)$, the right-hand-side of equation (10) is roughly approximated by Bo itself. This implies that the breakup thresholds in $G$ are characterized by the critical value of the Froude number $F r=W e /$ Bo around the unity.

\section{Conclusions}

Stability of bubbles subjected to oscillatory acceleration field has been studied experimentally both in micro and normal gravity environments. In the attempt to reveal the physical mechanism behind the breakup, the breakup threshold was determined and discussed in detail with accompanied observations on the bubble motion under the excitation. Determined critical parameters were correlated by similar power laws $K_{C} O h^{1.7} \Omega^{n}=c s t$ with the power $n$ around 1.9 in both environments. Confrontations with different possible breakup mechanisms were performed. The possible mechanisms due to the Rayleigh-Taylor instability and the resonance with bubble eigen-modes were shown to be inconsistent with the experiments. The inertial breakup mechanism similar to that of drops in a uniform flow was found to give consistent explanation to experimentally determined breakup thresholds. From an energetic point of view, a universal criterion for experiments in $G$ and $\mu G$ was proposed in terms of the Weber number $W e$. Calculated $W e$ values on the breakup thresholds with the help of the consideration on the bubble motion affected by fluid viscosity and capillarity showed the validity of the criterion in the experiments as seen in fig. 10 . This suggests that inertial flow perturbation in suspending fluid is responsable for the observed breakups. 
The authors gratefully thank D. Vallet for technical supports and $\mu G$ experimentation. The financial support from CNES (Centre National d'Etudes Spatiales) is acknowledged with appreciation.

\section{References}

1. M. Thiriet, Biology and Mechanics of Blood Flows: Part II: Mechanics and Medical Aspects (Springer, 2007)

2. H.R. Pruppacher, J.D. Klett, Microphysics of clouds and precipitation (Springer, 1997)

3. J. Fredsøe, R. Deigaard, Mechanics of coastal sediment transport (World scientific publishing company, 1992)

4. S.H. Davis, Annu. Rev. Fluid Mech. 8, 57 (1976)

5. J. Miles, D. Henderson, Annu. Rev. Fluid Mech. 22, 143 (1990)

6. F. Zoueshtiagh, S. Amiroudine, R. Narayanan, J. Fluid Mech. 628, 43 (2009)

7. A.V. Coward, D.T. Papageorgiou, IMA J. Appl. Math. 53(1), 75 (1994)

8. B. Thiria, S. Goujon-Durand, J.E. Wesfreid, J. Fluid Mech. 560, 123 (2006)

9. A.J. James, B. Vukasinovic, M.K. Smith, A. Glezer, J. Fluid Mech. 476, 1 (2003)

10. B. Vukasinovic, M.K. Smith, A. Glezer, Phys. Fluids 19, 012104 (2007)

11. J. Ellenberger, R. Krishna, Chem. Eng. Sci. 58, 705 (2003)

12. G.J. Jameson, J.F. Davidson, Chemical Engineering Science 21, 29 (1966)

13. F. Zoueshtiagh, H. Caps, M. Legendre, N. Vandewalle, P. Petitjeans, P. Kurowski, Eur. Phys. J. E 20(3), 317 (2006)

14. F. Zoueshtiagh, M. Legendre, H. Caps, N. Vandewalle, Phys. Fluids 16(9), S7 (2004)

15. R. Clift, J.R. Grace, M.E. Weber, Bubbles, Drops, and Particles, Dover 2005 edn. (Academic Press, Inc., New York, 1978)

16. R.M. Davies, S.G.I. Taylor, Proc. R. Soc. Ser. A 200(1062), 375 (1950)

17. P.G. de Gennes, F. Brochard-Wyart, D. Quéré, Gouttes, bulles, perles et ondes (Belin, 8, rue Férou 75278 Paris Cedex 06, 2002)

18. H. Yoshikawa, P. Kurowski, P. Petitjeans, F. Zoueshtiagh, H. Caps, Microgravity Sci. Technol. 19(3-4), 155 (2007)

19. F. Risso, Multiphase Science and technology 12, 1 (2000)

20. J.O. Hinze, AIChE J. 1, 289 (1955)

21. J.R. Grace, T. Wairegi, J. Brophy, Can. J. Chem. Eng. 56, 3 (1978)

22. C.E. Brennen, Fundamentals of Multiphase Flow (Cambridge University Press, New York, 2005)

23. M. Komabayashi, T. Gonda, K. K. Isono, Journ. Met. Soc. Japan 42(5), 330 (1964)

24. J. Kitscha, G. Kocamustafaogullari, Int. J. Multiphase Flow 15(4), 573 (1989)

25. H. Lamb, Hydrodynamics, 6th edn. (Cambridge Univ. Press, London, 1932)

26. A. Prosperetti, J. Fluid Mech. 100(2), 333 (1980)

27. G.I. Taylor, Tech. Rep. AC 10647/Phys. C69, Advisory Council on Scientific Research and Technical Development, Ministry of Supply (1949)

28. L.P. Hsiang, G.M. Faeth, Int. J. Multiphase Flow 21(4), $545(1995)$ 